References:

[1] Argent R, Patient Involvement With Home-Based Exercise Programs: Can Connected Health Interventions Influence Adherence? JMIR Mhealth Uhealth 2018.

[2] Lamb SE, Strengthening and Stretching for Rheumatoid Arthritis of the Hand Trial (SARAH).Lancet. 2015,385(9966).

[3] Azevedo R, Smartphone application for rheumatoid arthritis self-management: cross-sectional study revealed the usefulness, willingness to use and patients' needs. Rheumatol Int.2015, 35(10)

Disclosure of Interests: None declared

DOI: 10.1136/annrheumdis-2020-eular.5496

\section{AB1352-HPR SHARED CARE - AN ALTERNATIVE WAY TO COPE WITH INCREASING SERVICE DEMAND}

C. Yin Shuen ${ }^{1}$, N. Woon Leung2, L. Man Chi2, L. Chi Kwai2, C. Ching Man2, S. P. Kong2, L. Pik Fan2, C. L. Leung2. ${ }^{1}$ United Christian Hospital, Medicine and Geriatric, Hong Kong, Hong Kong (SAR); ${ }^{1}$ United Christian Hospital, Medicine and Geriatric, Hong Kong, Hong Kong (SAR)

Background: Rheumatic diseases are immune-mediated disorders that affect the musculoskeletal system, soft tissues, blood vessels and connective tissue. Patients with rheumatic diseases need regular follow up for disease and drug toxicity monitoring. To cope with the increasing service demand, the Division of Rheumatology in the United Christian Hospital developed and expanded the shared care service. In the conventional practice, patients have to been seen by rheumatologist for every visit while the shared care service involved follow up by rheumatologist and rheumatology nurse in alternate sessions.

Objectives:

1.To evaluate the effectiveness and safety of the shared cared service 2To evaluate the effectiveness of reduction in workload of rheumatology clinic Methods: This is a retrospective study involving the period from 1/1/2019 to $31 / 12 / 2019$. Patients who attended the rheumatology nurse clinic for shared care were recruited and reviewed. All patients were selected and referred by rheumatologists. Criteria for shared care included regular follow up in rheumatology clinic and stable clinical condition. The length of follow up is adjusted according to patient condition. Services provided by rheumatology nurse $(\mathrm{RhN})$ included disease education, drug and disease monitoring, drug advice and referral to othe professionals and community service as indicated. During each visit, patient's vital signs, disease activity and laboratory results were assessed according to standard protocol. RhN will make discharge record to ensure continuity of care. Results: Totally 489 episodes of attendance to nurse led clinic were recruited Majority (97.3\%) were arthritis patients. Others included lupus, vasculitis, Sjogren's syndrome and miscellaneous conditions. The length of follow up ranged from 3 weeks to 24 weeks and most of the patients were follow up between 8 to 16 weeks. Shared care patients included those with stable disease for interval monitoring, and patients for drug initiation and titration. The ratio for disease monitoring and drug monitoring are $41.3 \%$ and $58.7 \%$ respectively.

For the 489 episodes of attendance, $10(2 \%)$ episodes needed rheumatologist intervention and $8(1.6 \%)$ cases need advancement of follow up. Problems that required doctor interventions and advance follow up mainly are suboptimal disease control requiring medication adjustment or musculoskeletal ultrasound investigation. 178 (36.4\%) episodes of nursing intervention were delivered, majority were medication advice $(133,27.2 \%)$. Reasons for nursing intervention included adverse drug reaction, abnormal investigation results, poor drug adherence and disease flare up. There were no emergency department attendance or admission related to rheumatic problems within one month of $\mathrm{RhN}$ follow up.

Conclusion: The shared care service is smooth and can safely lengthen the follow-up intervals to reduce clinic visit burden in rheumatology clinic. RhN input also allowed prompt advice for steroid tapering and dose titration for disease specific medication for better disease control. Proper case selection and close collaboration between rheumatologist and rheumatology nurse is the key.

Disclosure of Interests: None declared

DOI: 10.1136/annrheumdis-2020-eular.4381

\section{HPR Professional education, training and competencies}

\section{AB1353-HPR EMPOWERING LEARNERS TO "OWN"THEIR PERFORMANCE: PRESENTING EDUCATIONAL PERFORMANCE DATA BACK TO LEARNERS AS A COMPONENT OF AND RATIONALE FOR SUBSEQUENT EDUCATION}

E. Johnson ${ }^{1}$, L. Eldasher ${ }^{2}$, D. Clausen ${ }^{3} \cdot{ }^{1} R M E I$ Medical Education, Outcomes \& Analytics, Voorhees Township, United States of America; ${ }^{2}$ RMEl Medical
Education, Medical Strategy, Voorhees, United States of America; ${ }^{3}$ RMEI Medical Education, Educational Strategy, Voorhees, United States of America

Background: Beginning in 2016, RMEl created multiple accredited online education focused on RA and designed for rheumatologists. In 2018, the educational scope expanded to include live meetings, starting with a symposium at EULAR. For al programming from 2016-18 (6 courses, in both live and online settings), educational content was developed based on performance data from the previous RA courses. During the 2019 EULAR symposium, we presented the outcomes findings - from both the 2018 symposium and online courses - to the assembled rheumatologists, identifying ongoing educational gaps observed in their specialty population. The rationale: to create continuity between symposia over time by demonstrating incremental improvements and continuing areas of need, while also endowing learners with a greater sense of ownership and investment in forthcoming educational content.

Objectives: To evaluate the impact of presenting learner's educational performance data to them before education addressing persistent gaps identified in their performance the year prior.

Methods: Data collected during the 2018 EULAR symposium was analyzed to understand the underlying drivers impacting poor performance in an identified area of ongoing educational need (cycling versus switching TNF inhibitors). A linear regression model was run including all non-related curriculum, demographic, and evaluation questions as possible drivers against those related low-scoring (at Post-Test) curriculum questions. The content of RMEl's 2019 symposium at EULAR was developed to address the identified significant drivers to improve population proficiency in cycling versus switching. In addition to developing content based on the above findings, that data was also presented to learners in poster format prior to their participation in the 2019 symposium. During the period between on-site registration and the start of the symposium, attendees had the opportunity to explore data-driven insights, via audio-guided posters located around the meeting room. These insights included discussion of 2018 data analysis, identified drivers of poor performance, introduced the iterative data-driven methodology employed, and rationale behind content development for the 2019 symposium. At the conclusion of the 2019 symposium learners were asked to describe the impact/relevance of being presented with their performance data, their intention of incorporating course content into practice, and what specifically they intended to change.

Results: Data was collected on 135 clinicians (primarily physicians who actively treat patients with rheumatoid arthritis) who attended, and participated in, the symposium. With specific regard to the impact of seeing their own data presented back to them, $80 \%$ reported that seeing the learner data from the 2018 symposium enhanced their current learning experience. Further, $86 \%$ reported that they intended to incorporate course content into their clinical practice. Specifically, this population reported an intent to change their treatment approach and patient education practices.

Conclusion: Education is only as effective as the degree to which the audience is engaged. While year over year data from 2016 through 2018 demonstrated that an iterative approach facilitated the meaningful and necessary reinforcement of challenging concepts, learners in prior years were not aware of the methodological underpinnings of the educational offerings. Presenting this population with findings derived from their performance - as a rationale for the education they were about to participate in proved a compelling motivator for active learner engagement, and may have had a positive influence on the degree to which learners implemented course content into their clinical practice.

Disclosure of Interests: None declared

DOI: 10.1136/annrheumdis-2020-eular.4748

\section{AB1354-HPR CURRENT SITUATION OF TRAINING IN RHEUMATOLOGY IN THE POSTGRADUATE CURRICULUMS OF INTERNAL MEDICINE, FAMILY MEDICINE, GERIATRICS, PAIN MEDICINE AND, PHYSICAL AND REHABILITATION MEDICINE ACROSS COLOMBIA}

D. G. Fernández-Ávila ${ }^{1}$, L. Patino ${ }^{2}$, J. Gutiérrez ${ }^{2} .{ }^{1}$ Pontificia Universidad Javeriana - Hospital Universitario San Ignacio., Bogotá, Colombia; ${ }^{2}$ Pontificia Universidad Javeriana - Hospital Universitario San Ignacio, Bogotá, Colombia

Background: Rheumatic diseases are an important complaint in patients although the incidence is low, they cause disability and impact on the health system. Currently, in Colombia, there are 198 rheumatologist, so it's important to know how is rheumatology training in specialties related to rheumatology, which are the specialties that the patient with a suspected rheumatic disease will visit at first.

Objectives: To describe the training status in rheumatology in the postgraduate curriculums of internal medicine, family medicine, geriatrics, pain medicine and, physical and rehabilitation medicine across Colombia.

Methods: This is a descriptive cross-sectional study. A survey was applied in each participating medical school using the RedCap $\AA$ platform. The questionnaire included multiple-choice responses and a textbox to complete. The survey was done to the 29 registered medical schools which offer the specializations 